\title{
Percolation, Perimetry, Planarity
}

Gady Kozma

\begin{abstract}
Let $G$ be a planar graph with polynomial growth and isoperimetric dimension bigger than 1 . Then the critical $p$ for Bernoulli percolation on $G$ satisfies $p_{c}<1$.
\end{abstract}

This short note relates to a famous problem first posed in the seminal paper of Benjamini and Schramm [1]: find some general conditions on a graph $G$ under which $p_{c}<1$. In particular they conjectured (question 2 ibid.) that if the isoperimetric dimension is $>1$ then this holds. The isoperimetric dimension is defined for an infinite connected graph $G$ by

$$
\operatorname{dim}(G):=\sup \left\{d \geq 1: \inf _{S} \frac{|\partial S|}{|S|^{(d-1) / d}}>0\right\}
$$

where the infimum is over all finite non-empty sets of vertices $S . \partial S$ is the set of edges with one vertex in $S$ and the other in $G \backslash S$.

A number of partial cases of this conjecture have been proved. In $[1$, theorem 2] it was proved that a positive Cheeger constant (i.e. $|\partial S|>c|S|$ ) implies $p_{c}<1$. In [4] this was proved for graphs which satisfy some complicated conditions on the geometry of the minimal cut-sets. Here we shall show this under one technical condition (polynomial growth) and one more essential condition (planarity).

Theorem. Let $G$ be a planar graph with no vertex accumulation points such that

1. There exist numbers $K$ and $D$ such that for all $v \in G$ and any $r \geq 1$ one has for the open ball $B(v, r)$ that the number of vertices satisfies $|B(v, r)| \leq K r^{D} ;$ and

2. There exist numbers $k, \epsilon>0$ such that for any finite non-empty set of vertices $S,|\partial S| \geq k|S|^{\epsilon}$.

Let $p_{c}$ be the critical $p$ for independent bond percolation on $G$. Then $p_{c}<1$.

2000 Mathematics Subject Classification: 60K35, 82B43, 05C10.

Keywords: Percolation, isoperimetric dimension, planar graph, duality. 
A vertex accumulation point is a point $x \in \mathbb{R}^{2}$ such that every neighborhood of $x$ contains infinitely many vertices of $G$. To be more precise, the theorem holds whenever the abstract planar graph $G$ has a plane representation $\Gamma$ with no vertex accumulation points. We assume $G$ has no loops or multiple edges.

Proof. Let us first dispose of uninteresting cases resulting from stating the theorem in too much generality. We may assume every vertex of $G$ has only finitely many neighbors since otherwise $p_{c}=0$. Further, By Wagner's theorem [8] a planar graph contains no more than a countable number of vertices with degree $\geq 3$. On the other hand, our graph $G$ cannot contain a component with all degrees $\leq 2$ since that would be a finite or infinite line and would violate the assumption that the isoperimetric dimension is $>1$. With Wagner's theorem we get that $G$ is countable. Hence the existence of an infinite cluster is a measurable event, $p_{c}$ is well defined and our theorem makes sense. This allows us to assume $G$ is connected by restricting to any infinite component.

We use Peierls argument, which states that $p_{c}<1$ whenever the number of minimal cut-sets of some vertex $v \in G$ of size $n$ is exponential (or less) in $n$. See e.g. [3, page 16]. Here a cut-set of $v$ is a set of edges $S$ such that $v$ is in a finite component of $G \backslash S$. The cut-set is minimal if every $S^{\prime} \varsubsetneqq S$ is not a cut-set. Since $G$ is planar we can define its dual graph $G^{*}$ by making any face of $G$ to be a vertex of $G^{*}$ and matching any edge $e$ of $G$ to an edge $e^{*}$ of $G^{*}$ between the two faces of $G$ on the two sides of $e$. Note that $G^{*}$ may contain loops and multiple edges. Formally we require the following from $G^{*}$ :

1. There is a one-to-one onto correspondence between $E(G)$, the edge set of $G$ and $E\left(G^{*}\right)$, which we denote by $*$.

2. A minimal cut-set of $G$ is carried by $*$ to a cycle (i.e. a simple closed path) of $G^{*}$ and vice versa.

The existence of $G^{*}$ is well known for finite graphs but for infinite graphs I was not able to find a completely suitable reference, hence we shall show the existence of $G^{*}$ in the appendix.

Thus we need to show that the number of cycles of length $n$ separating $v^{*}$ from infinity is $\leq C^{n}$ for some $n$. If, for example, $G^{*}$ happens to be a graph with bounded degree (as in [4]) we could have finished here. In general, however, $G^{*}$ could have unbounded degree and might not even be locally finite (a graph is locally finite if every vertex has finite degree).

It will be easier to examine simple paths. Let us therefore fix two vertices $a^{*} \neq b^{*} \in G^{*}$ and examine the number $p=p\left(a^{*}, b^{*} ; n\right)$ of simple paths of length $n$ starting from $a$ and ending at $b$. 
We shall now show that $p\left(a^{*}, b^{*} ; n\right) \leq C^{n}$ and then we shall be mostly finished. Let

$$
p(n):=\max _{a^{*} \neq b^{*} \in G^{*}} p\left(a^{*}, b^{*} ; n\right) .
$$

We wish to find an inequality connecting $p(n)$ and $p(2 n)$.

Let therefore $a^{*} \neq b^{*} \in G^{*}$ and let $\gamma^{*}$ be one simple path of length $2 n$ between $a^{*}$ and $b^{*}$ which we will use as a reference. Let $\delta^{*}$ be a second such path — we wish to bound the number of possibilities for $\delta^{*}$. For every edge $e^{*}$ of $\gamma^{*}$ there are two vertices of $G$ adjacent to $e$-let $\left\{v_{1}, \ldots, v_{4 n}\right\}$ be the complete list. Let $f^{*}$ be the $n$ 'th edge of $\delta^{*}$, and let $w_{1}, w_{2}$ be the two vertices of $G$ on both sides of $f$. Since $\gamma^{*} \cup \delta^{*}$ is a closed path, either $f^{*} \in \gamma^{*}$ or there exists a cycle $\beta^{*} \subset \gamma^{*} \cup \delta^{*}$ containing $f^{*}$. Assume the second. Since $\beta$ is a minimal cut-set in $G$ we have that it is the boundary of some finite connected set $Q$ and therefore one of the $w_{j}$-s is in $Q$. Denote it by $w$. It is not possible for $\beta^{*} \subset \delta^{*}$ (since $\delta^{*}$ is simple) so there exists at least one of the $\left\{v_{1}, \ldots, v_{4 n}\right\}$ which is in $Q$. Denote it by $v$. Since $|\partial Q|=|\beta| \leq\left|\gamma^{*} \cup \delta^{*}\right| \leq 4 n$ then by the isoperimetric inequality we see that $|Q| \leq(4 n / k)^{1 / \epsilon}$. Since $Q$ is connected the distance in $G$ between $v$ and $w$ must be $\leq(4 n / k)^{1 / \epsilon}$. We think about this as $w \in \bigcup_{i} B\left(v_{i},(4 n / k)^{1 / \epsilon}\right)$ and we have also subsumed the case that $f^{*} \in \gamma^{*}$ which wasn't covered by the argument above (choose $w$ from $w_{1}, w_{2}$ arbitrarily in this case). Polynomial growth shows that $w$ has $\leq 4 n K(4 n / k)^{D / \epsilon}$ possibilities. Using the polynomial growth inequality with $r=2$ we know that every vertex of $G$ has no more than $K 2^{D}$ adjacent edges, so $f$ has $\leq 4 n K^{2}(8 n / k)^{D / \epsilon}$ possibilities and the same holds (with another factor of 2 ) for the middle vertex of $\delta^{*}$. Since this holds for every $\delta^{*}$ we are left with the inequality

$$
p(2 n) \leq 8 n K^{2}(8 n / k)^{D / \epsilon} p(n)^{2} .
$$

An identical argument shows that $p(1) \leq(2 / k)^{1 / \epsilon}$ and from here a simple induction shows that $p\left(2^{n}\right) \leq \exp \left(C 2^{n}\right)$ where $C=C(k, K, \epsilon, D)$ is independent of $n$. This obviously implies $p(n) \leq C^{n}$ for some other $C$.

To finish the theorem, let $v \in G$ and examine a minimal cut-set $\gamma$ of size $n$. Since $\gamma$ is the boundary of the component of $G \backslash \gamma$ containing $v$, we see that this component has size $\leq(n / k)^{1 / \epsilon}$ and in particular it is contained in $B\left(v,(n / k)^{1 / \epsilon}\right)$. By polynomial growth we see that there are no more than $K(n / k)^{D / \epsilon}$ possible vertices in the component and hence no more than $K^{2}(2 n / k)^{D / \epsilon}$ edges which may participate in the cut-set. For each of these edges $e$, a cut-set containing $e$ is, viewed in $G^{*}, e^{*} \cup\{$ an open simple path of length $n-1\}$. Therefore the number of possibilities for a minimal cut-set is

$$
\leq K^{2}(2 n / k)^{D / \epsilon} p(n-1) \leq C^{n}
$$

for some $C$. This proves the theorem. 


\section{Appendix: duality}

Duality of infinite planar graphs was investigated by Thomassen $[6,7]$ and recently by Bruhn and Diestel [2] but we cannot use either for the following reason. Both define the dual (denote it by $\widehat{G}$ to differentiate) such that a cycle in $\widehat{G}$ corresponds to a bond in $G$ where a bond is a minimal set dividing $G$ into components but without requiring any one of them to be finite. Take as an example $G$ to be an infinite bi-directional line. $G^{*}$ (which is, in this case, uniquely defined up to the addition of isolated vertices) is a graph with two vertices connected by infinitely many edges, while $\widehat{G}$ is a single vertex with infinitely many loops. Hence our minimal cut-sets correspond in $\widehat{G}$ to a not-necessarily simple closed path and the proof does not go through.

It is quite likely that the condition that the graph has no accumulation points is redundant (with the same proof structure). Condition (2) in the definition of $G^{*}$ must be relaxed by removing the "vice versa" claim, but this can be worked around in the proof of the theorem. Hence the only obstacle is the existence of $G^{*}$. It would be interesting to show that $G^{*}$ exists under, say, the condition $(*)$ of $[2]$.

Lemma. Let $G$ be a connected locally finite planar graph with no accumulation points. Then a $G^{*}$ satisfying the requirements (1) and (2) exists.

Proof sketch. By [5, theorem 3] there exists a straight line triangulation $\Delta$ such that $G$ is a subgraph of $\Delta$. Define two triangles of $\Delta$ to neighbor if they have a common edge and it is not in $G$. We will call the components of the neighborhood graph on the triangles of $\Delta$ "faces of $G$ " and they will serve as the vertex set for $G^{*}$. For every edge $e$ of $G$ we will define the edge $e^{*}$ to be adjacent to the faces containing its two neighboring triangles (which may be the same). Hence we need only show the relation between minimal cut-sets of $G$ and cycles of $G^{*}$.

To show this we will construct $G^{*}$ as a geometric dual of $G$. In any face $f$ of $G$ pick an arbitrary edge $e$ in $\partial f$, let $T$ be the triangle of $f$ containing $e$ and let $p \in \mathbb{R}^{2}$ be the middle $T$. Now trace around $\partial f$ starting from $T$ and $e$ using the triangulation structure of $\Delta$ i.e. at any vertex turn around until hitting an edge of $G$ and continue to the appropriate triangle. This process reaches the entire boundary of $f$. To see this, use the tracing process to construct a simple path $\rho \subset \mathbb{R}^{2}$ ( $\partial f$ need not be simple) slightly inside $f$ (e.g. in every triangle $S$ of $\Delta$ participating in the tracing process make $\rho$ go through the third of $S$ closest to $G$ ). If the resulting $\rho$ is infinite it must go to infinity (because $G$ has no accumulation points) and we can add the point at infinity to close it. Let now $e^{\prime} \in \partial f$ be arbitrary and we wish to show that it 
participated in the tracing process. Let $S_{1}, \ldots, S_{n}$ be a path of neighboring triangles such that $e^{\prime} \in \partial S_{1}$ and $S_{n}=T$. Let $\sigma \subset \mathbb{R}^{2}$ be a path starting in the middle of $e^{\prime}$, linear in every $S_{i}$ and passing through the middle of the edge joining $S_{i}$ and $S_{i+1}$, and finally ending in $p$. We use Jordan's theorem with $\rho$ and get two components $A$ and $B$ and examining $T$ it is easy to see that $p$ is in one (say $A$ ) while $e$ (and therefore, by connectivity, of all $G$ ) is in the other. Therefore $\rho \cap \sigma \neq \emptyset$. But by definition this can only happen in $S_{1}$ and therefore $\rho$ passed through $S_{1}$ and therefore $e^{\prime}$ participated in the tracing process.

The tracing process allows to connect $p$ to the middle of any $e^{\prime} \in \partial f$ by disjoint curves. These curves will be "half edges", the other half coming from the other face. Hence we have completed a description of $G^{*}$ as a planar graph. Further, $e \cap f^{*} \neq \emptyset$ iff $e=f$ and then they intersect at a single point and are transversal at that point. This immediately shows that any cycle $\gamma^{*}$ in $G^{*}$ maps to a cut in $G-$ Jordan's theorem shows that the set $Q$ of vertices of $G$ inside $\gamma^{*}$ is disjoint from the ones outside $\gamma^{*}$, and the transversality shows that for every $e^{*} \in \gamma^{*}$ exactly one of the vertices adjacent to $e$ is in $Q$, so $Q \neq \emptyset$. The fact that there are no accumulation points in $G$ shows that $Q$ is finite and hence $\gamma$ is a cut.

On the other hand, if $\gamma$ is a minimal cut then every vertex in $\gamma^{*}$ must have degree at least 2. To see this take one $e^{*} \in \gamma^{*}$ and let $p^{*}$ be an adjacent face of $G$, and assume no other $f^{*} \in \gamma^{*}$ is adjacent to $p^{*}$. Let $\delta$ be the boundary of $p^{*}$ which is either a closed path or a bi-directionally infinite path in $G$ (in neither case necessarily simple). If $\delta$ is finite then it forms a path in $G \backslash \gamma$ between the two ends of $e$, in contradiction of the minimality of $\gamma$. If $\delta$ is infinite then both ends of $e$ are connected to an infinite number of vertices in $G$, in contradiction to the fact that one side must be finite.

Now, a graph $\gamma^{*}$ with minimal degree $\geq 2$ must contain a cycle, $\delta^{*}$. By the previous argument, $\delta$ would be a cut. But since we assumed $\gamma$ is minimal, we must have $\gamma=\delta$ and hence $\gamma^{*}$ is a cycle. Conversely, if $\gamma^{*}$ is a cycle then $\gamma$ is a cut. If $\delta \subset \gamma$ is minimal then $\delta^{*} \subset \gamma^{*}$ is a cycle. But cycles don't contain subcycles so $\delta^{*}=\gamma^{*}$ and we have established both directions of the correspondence between minimal cuts of $G$ and cycles of $G^{*}$, and we are done.

Acknowledgments. I wish to thank Itai Benjamini for many fruitful discussions. Gábor Pete, Paul Seymour and Carsten Thomassen helped with references and advice. 


\section{References}

[1] Benjamini, I. And Schramm, O.: Percolation beyond $\mathbb{Z}^{d}$, many questions and a few answers. Electron. Comm. Probab. 1 (1996), no. 8, 71-82.

[2] Bruhn, H. And Diestel, R.: Duality in infinite graphs. Combin. Probab. Comput. 15 (2006), no. 1-2, 75-90.

[3] Grimmetr, G.: Percolation. Second edition. Grundlehren der Mathematischen Wissenschaften [Fundamental Principles of Mathematical Sciences] 321. Springer-Verlag, Berlin, 1999.

[4] Procacci, A. And Scoppola, B.: Infinite graphs with a nontrivial bond percolation threshold: some sufficient conditions. J. Statist. Phys. 115 (2004), no. 3-4, 1113-1127.

[5] Thomassen, C.: Straight line representations of infinite planar graphs. J. London Math. Soc. (2) 16 (1977), no. 3, 411-423.

[6] Thomassen, C.: Planarity and duality of finite and infinite graphs. J. Combin. Theory Ser. B 29 (1980), no. 2, 244-271.

[7] Thomassen, C.: Duality of infinite graphs. J. Combin. Theory Ser. B 33 (1982), no. 2, 137-160.

[8] Wagner, K.: Fastplättbare Graphen. (German). J. Combinatorial Theory 3 (1967), 326-365.

Recibido: 7 de octubre de 2005

Revisado: 23 de marzo de 2006

Gady Kozma

The Weizmann Institute of Science

Rehovot 76100, Israel

gady. kozma@weizmann.ac.il 